\title{
Primary Cutaneous B-Cell Lymphomas in Patients With Impaired Immunity
}

\author{
Irene Russo, Laura Fagotto, Alvise Sernicola and Mauro Alaibac* \\ Unit of Dermatology, University of Padua, Padua, Italy
}

Keywords: cutaneous B-cell lymphoma, immunosuppression, posttransplant lymphoproliferative disorder, EBV, HIV

Primary cutaneous B-cell lymphomas (CBCL) are a group of non-Hodgkin lymphomas (NHL) defined by a clonal B-cell proliferation involving the skin and no extracutaneous districts and constitute $20-25 \%$ of primary cutaneous lymphomas (1). The "2018 update of the WHO-EORTC classification” recognized three subtypes: primary cutaneous marginal zone lymphoma (PCMZL), primary cutaneous follicle center cell lymphoma (PCFCCL), and primary cutaneous diffuse large B-cell lymphoma, leg type (PCDLBCL, LT) (2). A diagnosis of PCDLBCL, NOS, covers rare cases that are not classifiable as either PCDLBCL, LT or PCFCL.

PCMZL is an indolent form of lymphoma that accounts for up to $30-40 \%$ of primary cutaneous lymphomas. PCMZL tends to relapse in the skin, but dissemination to extracutaneous sites is rare and the prognosis is excellent, with an estimated five year survival rate of 99\% (3). PCFCCL is an indolent lymphoma that comprehends $\sim 40-50 \%$ of primary CBCLs. PCFCCLs can recur in up to $30 \%$ of patients, but dissemination to extracutaneous sites is rare and survival is over $95 \%$ at 5 years (2). PCMZL and PCFCCL are characterized by an indolent behavior; but PCDLBCL, LT is instead characterized by an intermediate to aggressive clinical behavior. PCDBCL, LT occurs most commonly with skin lesions on the lower legs, accounts for under $20 \%$ of primary CBCL and affects elderly, predominantly female patients. Compared to the other subtypes of CBCL, this lymphoma disseminates to extracutaneous sites more often and has a more unfavorable prognosis, with a 5 year survival rate of around $55 \%$.

$\mathrm{EBV}+$ mucocutaneous ulcer (EBVMCU) is a provisional type of indolent B-cell lymphoma that occurs in cases of immunosuppression due to treatment or age (4). CBCL are rare entities in both immunocompetent and immunosuppressed patients. However, these two groups of patients show different subtypes, with different clinical behavior and prognosis. In immunocompetent patients primary CBCL usually have an indolent behavior is typical of PCMZL and PCFCCL. They also have a favorable outcome, with survival rates above 95\% after 5 years. Aggressive subtypes are very rare in this group. Even if immunosuppression is a known risk factor in lymphoproliferative disorders, it is difficult to compare the different causes of immunosuppression. However, in all types of immunosuppression, aggressive subtypes of CBCL are observed more frequently than in immunocompetent patients, while indolent subtypes are virtually non-existent $(5,6)$.

We focused on CBCL in two main contexts of immunosuppression, iatrogenic and noniatrogenic, to highlight the role of immune surveillance in determining the behavior of these disorders. Different subtypes of CBCL are associated to different prognosis, which could be due to how CBCL interact with the immune system and whether it is functioning or impaired.

Although epidemiological data on primary cutaneous lymphomas in immunocompromised patients is scarce, there are case series and isolated reports on HIV and EBV infections in some congenital immunodeficiency disorders and in iatrogenic immunosuppression. Non-iatrogenic immunosuppression may occur when infectious agents impair host immune response. The immunological events induced by these agents, notably EBV, HIV and Borrelia (6-8), are 
responsible for different types of immune dysregulation. Even though it is difficult to compare these different types of immune dysregulation, this overall response provides insight into the role of an efficient immune system in the surveillance for malignant cells and oncogenic pathogens.

\section{NON-IATROGENIC IMMUNOSUPPRESSION}

\section{CBCL and EBV Infection}

EBV is a ubiquitous virus, capable of establishing a persistent infection that remains subclinical in the majority of individuals. This viral latency involves a complex homeostasis in the host, created by the pathogen's mechanisms of immune and host surveillance evasion (7). EBV selectively colonizes host Bcells and is able to persist in EBV+ memory B-cells, which become the viral reservoir. In the initial form of latency infected B-cells display a growth-transformation phenotype. They also express a wide array of EBV latent proteins, leading to immune recognition by cytotoxic T-cells. Viral latent membrane proteins provide the infected B-cells with anti-apoptotic and proliferative signals, causing cell immortalization and increasing the risk of developing malignant B-cell proliferation (9). In immunocompetent carriers, the effective immune clearance of EBV-infected cells leads to forms of deeper latency that also evade host surveillance, meaning EBV is a silent infection. Ineffective viral clearance is common when immunity is suppressed due to concurrent infection, iatrogenic causes, and mutations responsible for the syndrome.

Specific types of primary immunodeficiency suggest potential strategies to treat EBV-induced conditions, as they provide unique models for understanding the immune mechanisms required to control EBV infection. Abnormal susceptibility to EBV is associated to X-linked lymphoproliferative disease (10), Wiskott-Aldrich syndrome (11) and different defects responsible for combined immunodeficiency. Absence of normal T-cell function in severe combined immunodeficiency exposes patients to EBV-induced B-cell lymphoproliferation at an early age (12). On the other hand, common variable immunodeficiency (CVID) causes B-cell defects that prevent plasma cell differentiation and antibody secretion together with variable defects in T-cells and dendritic cells.

Though prominent EBV infections are not typical of CVID, increased risk of malignancy due to B-cell lymphoma is occasionally associated with EBV (13). EBV-associated lymphoproliferations rarely present to the skin. Cases of CBCL have been reported in the setting of iatrogenic immunosuppression and DLBCL, NOS, is the most common subtype. These cases are associated to prolonged administration of drugs, leading to years of immune dysregulation before the onset of CBCL (14).

In subjects with no known cause of immune suppression, an association with EBV has been demonstrated in rare types of CBCL, with positive in situ hybridization of EBV. Primary cutaneous EBV + DLBCL is an aggressive B-cell lymphoma that has been reported in immunocompetent adults over 50 years old, and is associated with a poor prognosis. The expression of latent membrane proteins is associated to clonal B-cell proliferation, suggesting that these proteins have a role in $\mathrm{B}$ cell immortalization (9). HHV-8, a gamma herpesvirus related to $\mathrm{EBV}$, is capable of infecting B-cells and plasmablasts through virally secreted proteins which can stimulate proliferation of Bcells and plasma cells. In certain cases, HHV-8 induces a Bcell plasmablastic lymphoma, a rare subtype of DLBCL $(15,16)$. Cutaneous lymphomagenesis is related to HHV-8 infection and occurs in a setting of immunodeficiency in this variant, as well as in specific presentations of primary effusion lymphoma in the $\operatorname{skin}(17,18)$.

\section{CBCL and HIV Infection}

Lymphomas associated with HIV infection usually share advanced-stage presentation and an aggressive clinical course. Prior to the introduction of combination antiretroviral therapy (cART) the risk of NHL was over 100 times higher in AIDS patients. Following the introduction of cART, the relative risk is still around 10-20. The most aggressive subtypes, DLBCL and $\mathrm{BL}$, remain common and are associated to a significantly reduced survival rate when compared to HIV-negative patients with the same diagnosis (6). Primary cutaneous lymphomas represent $10 \%$ of extranodal NHLs and have a B-cell origin in $15 \%$ of HIV patients. It has been reported that cases of isolated skin disease with no evidence of other localizations respond to cART alone, highlighting the role of immune reconstitution in the control of CBCL (19).

\section{CBCL and Borrelia}

Persistent infection with Borrelia burgdorferi $(B b)$ and protozoal species of Leishmania may be the consequence of pathogeninduced immunosuppression $(20,21)$. The mechanisms of immunotolerance have been studied in murine models of $B b$ infection and include inhibition of T-cell activation and of effective antibody mediated response $(8,22) . B b$ infection has been associated with PCMZL in some European cases (23). Lymphomagenesis in the skin could be triggered by pathogen-induced immunosuppression or alternatively, by chronic inflammatory stimulation and persistent lymphocyte activation due to an inability to eliminate the pathogen (24).

In the late stage of Lyme borreliosis, lymphocyte infiltration in the dermis creates a "lymphocytoma" or cutaneous Bcell pseudolymphoma (24). As the histology of B-cell pseudolymphoma and PCMZL are similar (4, 25), some authors suggest that there is a progression from lymphocitoma and pseudolymphoma to clonal proliferation that conducts to PCMZL (24). The monoclonality of B-cells is a useful way to differentiate between pseudolymphoma and lymphoma (24). The association between $B b$ and PCMZL is supported by the remission of the disease after antibiotic therapy is used to treat the $B b$ infection. Despite the fact that many patients do not respond to this treatment, in some cases antibiotic therapy has led to the remission of $B b$ positive PCMZL $(24,25)$. Taking into account the indolent behavior of PCMZL, attempts to treat $B b$ positive PCMZL with antibiotics should be considered as a first line therapy (25). The indolent behavior of CBCL, as observed 
in relation to $B b$ infection, supports the hypothesis that chronic antigenic stimulation is determined by the bacteria, which is in this case, the main mechanism causing lymphomagenesis. In chronic leishmaniosis, variable parasite-host interactions are responsible for a spectrum of cutaneous syndromes that may induce B-cell proliferation. However, bacterial association with CBCL has not as yet been clearly defined (26).

\section{IATROGENIC IMMUNOSUPPRESSION CBCL and Transplant Patients}

Iatrogenic immunosuppression is usually observed in transplant patients and is related to anti-rejection therapy. The risk of developing any kind of NHL increases over six-fold following organ transplantation and varies greatly according to subtypes. This increased risk of post-transplant lymphoproliferative disorders (PTLD) involves a specific spectrum of histologic subtypes and of associated risk factors. Aggressive subtypes of PTLD show strong elevations of risk, while only few cases of indolent lymphomas are reported in transplant recipients with no significant elevation in risk (27).

Extranodal disease is a common manifestation in these patients and the skin is a major localization (28). Seckin et al. (5) collected a multicenter European retrospective of primary cutaneous PTLD, of which $31.4 \%$ were primary CBCL. Of these primary CBCL, $90.9 \%$ were associated with EBV. The remaining 9.1\% DLBCL, NOS were EBV-negative. In terms of patients, $27.3 \%$ from this series had a fatal outcome due to PTLD, which is exceptional considering that the prognosis of CBCL is more favorable than that of systemic forms. Risk factors associated to cutaneous B-cell PTLD are anti-rejection therapy and EBV infection. Different drug regimens influence the risk of PTLD.

Heart transplant recipients that require a high dose of immunosuppressive therapy were overrepresented in published cases, accounting for $36.4 \%$ of patients with B-cell PTLD (5). The association between EBV and B-cell PTLD is well established,

\section{REFERENCES}

1. La Selva R, Alberti Violetti S, Delfino C, Grandi V, Cicchelli S, Tomasini C, et al. A literature revision in primary cutaneous B-cellm lymphoma. Indian J Dermatol. (2017) 62:146-57. doi: 10.4103/ijd.IJD_74_17

2. Willemze R, Cerroni L, Kempf W, Berti E, Facchetti F, Swerdlow SH, et al. The 2018 update of the WHO-EORTC classification for primary cutaneous lymphomas. Blood. (2019) 133:1703-14. doi: 10.1182/blood-2018-11-881268

3. Willemze R, Jaffe ES, Burg G, Cerroni L, Berti E, Swerdlow SH, et al. WHOEORTC classification for cutaneous lymphomas. Blood. (2005) 105:376885. doi: 10.1182/blood-2004-09-3502

4. Kempf W, Zimmermann AK, Mitteldorf C. Cutaneous lymphomas-an update 2019. Hematol Oncol. (2019) 37:43-47. doi: 10.1002/hon.2584

5. Seçkin D, Barete S, Euvrard S, Francès C, Kanitakis J, Geusau A, et al. Primary cutaneous posttransplant lymphoproliferative disorders in solid organ transplant recipients: a multicenter European case series. Am J Transplant. (2013) 13:2146-53. doi: 10.1111/ajt.12281

6. Re A, Cattaneo C, Rossi G. HIV and lymphoma: from epidemiology to clinical management. Mediterr J Hematol Infect Dis. (2019) 11:e2019004. doi: 10.4084/mjhid.2019.004

7. Tangye SG, Palendira U, Edwards ESJ. Human immunity against EBV-lessons from the clinic. J Exp Med. (2017) 214:269-83. doi: 10.1084/jem.20161846 with an estimated $50-70 \%$ rate of EBV-positivity discovered with FISH or PCR in tumor specimens (28). Chronic immune suppression impairs T-cell surveillance of EBV-infected B-cells and leads to the uncontrolled proliferation of EBV-transformed B-cells. This process also occurs in EBVMCU associated to iatrogenic immunosuppression. The reduction of therapy may be sufficient to achieve remission of this cutaneous EBV-related disorder (29). Several cases of aggressive subtypes of cutaneous B-cell PTLD have been reported, while indolent subtypes have not been observed in transplant recipients (5). The absence of the indolent forms of CBCL (PCMZL and PCFCCL) in this group of patients indicates that the development of these subtypes is probably hampered by immunosuppressive regimen. An analogous disproportion between aggressive and indolent forms of primary cutaneous lymphoma in immunocompromised patients, has been previously discussed in PTLD of T-cell origin (30).

\section{CONCLUSIONS}

In conclusion, these observations in immunosuppressed patients highlight the difference between indolent and aggressive forms of CBCL. Indolent subtypes are generally not observed in immunocompromised subjects. This finding, together with the clinical behavior of indolent forms in immunocompetent subjects, suggests that these lymphoproliferative processes are induced by mechanisms closely related to chronic inflammation. On the other hand, aggressive forms of CBCL are generally observed in the context of immunosuppression, where they are favored by altered immune surveillance and oncogenic viral stimuli, and should be regarded as true lymphomas.

\section{AUTHOR CONTRIBUTIONS}

All authors listed have made a substantial, direct and intellectual contribution to the work, and approved it for publication.

8. Tracy KE, Baumgarth N. Borrelia burgdorferi manipulates innate and adaptive immunity to establish persistence in rodent reservoir hosts. Front Immunol. (2017) 8:116. doi: 10.3389/fimmu.2017. 00116

9. Tokuda Y, Fukushima M, Nakazawa K, Oguchi S, Koganehira Y, Yamaura $\mathrm{M}$, et al. A case of primary Epstein-Barr virus-associated cutaneous diffuse large B-cell lymphoma unassociated with iatrogenic or endogenous immune dysregulation. J Cutan Pathol. (2008) 35:666-71. doi: 10.1111/j.1600-0560.2007.00859.x

10. Parolini S, Bottino C, Falco M, Augugliaro R, Giliani S, Franceschini R, et al. X-linked lymphoproliferative disease: 2B4 molecules displaying inhibitory rather than activating function are responsible for the inability of natural killer cells to kill Epstein-Barr virus-infected cells. J Exp Med. (2000) 192:33746. doi: 10.1084/jem.192.3.337

11. Albert MH, Bittner TC, Nonoyama S, Notarangelo LD, Burns S, Imai K, et al. X-linked thrombocytopenia (XLT) due to WAS mutations: clinical characteristics, long-term outcome, and treatment options. Blood. (2010) 115:3231-8. doi: 10.1182/blood-2009-09-239087

12. Hauck F, Voss R, Urban C, Seidel MG. Intrinsic and extrinsic causes of malignancies in patients with primary immunodeficiency disorders. J Allergy Clin Immunol. (2018) 141:59-68.e4. doi: 10.1016/j.jaci.2017. 06.009 
13. Bethune CA, Gompels MM, Taylor C, Angus B, Spickett GP. Treatment of EBV driven lymphoproliferation with erythrophagocytosis: 12 year follow up. J Clin Pathol. (2001) 54:328-31. doi: 10.1136/jcp.54.4.328

14. Verma S, Frambach GE, Seilstad KH, Nuovo G, Porcu P, Magro CM. EpsteinBarr virus-associated B-cell lymphoma in the setting of iatrogenic immune dysregulation presenting initially in the skin. J Cutan Pathol. (2005) 32:47483. doi: 10.1111/j.0303-6987.2005.00363.x

15. Dupin N, Diss TL, Kellam P, Tulliez M, Du MQ, Sicard D, et al. HHV8 is associated with a plasmablastic variant of Castleman disease that is linked to HHV-8-positive plasmablastic lymphoma. Blood. (2000) 95:140612. doi: 10.1182/blood.V95.4.1406.004k26_1406_1412

16. Kempf W, Kazakov D V., Mitteldorf C. Cutaneous lymphomas: an update. Part 2: B-cell lymphomas and related conditions. Am J Dermatopathol. (2014) 36:197-210. doi: 10.1097/DAD.0b013e318289b20e

17. Verma S, Nuovo GJ, Porcu P, Baiocchi RA, Crowson AN, Magro CM. Epstein-Barr virus- and human herpesvirus 8-associated primary cutaneous plasmablastic lymphoma in the setting of renal transplantation. J Cutan Pathol. (2005) 32:35-9. doi: 10.1111/j.0303-6987.2005.00258.x

18. Crane GM, Xian RR, Burns KH, Borowitz MJ, Duffield AS, Taube JM. Primary effusion lymphoma presenting as a cutaneous intravascular lymphoma. $J$ Cutan Pathol. (2014) 41:928-35. doi: 10.1111/cup.12405

19. Villafañe MF, Corti M. Primary cutaneous b-cell lymphoma successfully treated with highly active antiretroviral therapy alone: a case report and review of the literature. Indian J Dermatol. (2011) 56:418-20. doi: 10.4103/0019-5154.84744

20. Diterich I, Rauter C, Kirschning CJ, Hartung T. Borrelia burgdorferi-induced tolerance as a model of persistence via immunosuppression. Infect Immun. (2003) 71:3979-87. doi: 10.1128/IAI.71.7.3979-3987.2003

21. Rodrigues V, Cordeiro-Da-Silva A, Laforge M, Silvestre R, Estaquier J. Regulation of immunity during visceral Leishmania infection. Parasites and Vectors. (2016) 9:1-13. doi: 10.1186/s13071-016-1 412-x

22. Elsner RA, Hastey CJ, Olsen KJ, Baumgarth N. Suppression of long-lived humoral immunity following borrelia burgdorferi infection. PLoS Pathog. (2015) 11:e1004976. doi: 10.1371/journal.ppat.1004976

23. Cerroni L, Zöchling N, Pütz B, Kerl H. Infection by Borrelia burgdorferi and cutaneous B-cell lymphoma. J Cutan Pathol. (1997) 24:457-61. doi: 10.1111/j.1600-0560.1997.tb01318.x
24. Perrone S, D’Elia GM, Annechini G, Pulsoni A. Infectious aetiology of marginal zone lymphoma and role of anti-infective therapy. Mediterr $J$ Hematol Infect Dis. (2016) 8:1-13. doi: 10.4084/mjhid.2016.006

25. Ponzoni M, Ferreri AJM. Bacteria associated with marginal zone lymphomas. Best Pract Res Clin Haematol. (2017) 30:3240. doi: 10.1016/j.beha.2017.01.001

26. Yavuzer R, Akyürek N, Özmen S, Demirtaş Y, Ataoglu O. Leishmania cutis with b-cell cutaneous hyperplasia [7]. Plast Reconstr Surg. (2001) 108:21778. doi: 10.1097/00006534-200112000-00088

27. Clarke CA, Morton LM, Lynch C, Pfeiffer RM, Hall EC, Gibson TM, et al. Risk of lymphoma subtypes after solid organ transplantation in the United States. Br J Cancer. (2013) 109:280-8. doi: 10.1038/bjc.2013.294

28. Jagadeesh D, Woda BA, Draper J, Evens AM. Post transplant lymphoproliferative disorders: risk, classification, and therapeutic recommendations. Curr Treat Options Oncol. (2012) 13:12236. doi: 10.1007/s11864-011-0177-x

29. Dojcinov SD, Venkataraman G, Raffeld M, Pittaluga S, Jaffe ES. EBV positive mucocutaneous ulcer-a study of 26 cases associated with various sources of immunosuppression. Am J Surg Pathol. (2010) 34:40517. doi: 10.1097/PAS.0b013e3181cf8622

30. Ferranti M, Tadiotto Cicogna G, Russo I, Alaibac M. Immunomodulation in cutaneous t cell lymphoma. Front Oncol. (2019) 9:1025. doi: 10.3389/fonc.2019.01025

Conflict of Interest: The authors declare that the research was conducted in the absence of any commercial or financial relationships that could be construed as a potential conflict of interest.

The reviewer EB declared a past co-authorship with one of the authors MA to the handling editor.

Copyright (c) 2020 Russo, Fagotto, Sernicola and Alaibac. This is an open-access article distributed under the terms of the Creative Commons Attribution License (CC BY). The use, distribution or reproduction in other forums is permitted, provided the original author(s) and the copyright owner(s) are credited and that the original publication in this journal is cited, in accordance with accepted academic practice. No use, distribution or reproduction is permitted which does not comply with these terms. 